\title{
Aplicación del modelo Investigación Apreciativa al estudio de usuarios en la creación de una biblioteca especializada en la piedra natural
}

\author{
Applying Appreciative Inquiry Theory to the user studies design \\ in creating a library specialized in the natural stone
}

\author{
Carmen Gómez Camarero y Rocío Palomares Perrault \\ Dpto. de Filología Griega, Estudios Árabes, Lingüística General y Documentación, \\ Facultad de Filosofía y Letras, Universidad de Málaga, España
}

\begin{abstract}
Resumen
El objetivo de nuestro trabajo es ofrecer una propuesta metodológica en la fase de estudio de necesidades de información de usuarios de una biblioteca especializada en la piedra natural. Concretamente, nos referimos a la aplicación del modelo teórico Investigación Apreciativa (IA), ideado por D. Cooperrider y S. Srivastva (1986), un nuevo paradigma que se centra en el núcleo positivo de una organización o sistema, en el apoyo de los puntos fuertes y talentos de los individuos que conforman las organizaciones. Desde esta perspectiva el grupo de investigación que tiene como objetivo la planificación y diseño de una biblioteca especializada en la piedra natural (Biblioteca TinoStone), se dispone a conocer las necesidades de los potenciales usuarios de la misma. Para ello, se sirve de metodologías de recopilación de datos mixtas (cuestionarios, entrevistas) pero la novedad estriba en el cómo se realizan esas entrevistas: se invita a los usuarios a imaginar, a diseñar y a crear, de forma colaborativa, la biblioteca "ideal" para compartir e implementar conocimiento de una manera intencional y activa. Es una búsqueda cooperativa de lo mejor que existe en las personas y en sus organizaciones, evitando en todo momento la negatividad y la crítica.
\end{abstract}

Palabras clave: Investigación apreciativa. Estudios de usuarios. Biblioteca de empresas.

\section{Introducción}

La multinacional TINO Stone Group, dedicada a la extracción, tratamiento y comercialización de la piedra natural, encomienda al Grupo de Investigación de documentación de la Universidad e Málaga (UMA), el diseño y planificación de una biblioteca física y virtual especializada sobre la piedra natural con el objeto de convertirse en el centro de referencia en el estudio de la piedra natural en el mundo (Goodwill, 2005). Con este propósito el Grupo de Investigación diseña un proyecto dividido, a su vez en tres sub-proyectos, es decir:

\begin{abstract}
The aim of our study is to offer a methodological propose in studying the users' information needs for a library specialized in the natural stone. More precisely, we are referring to the implementation of the theoretical model Appreciative Inquiry (AI), articulated by D. Cooperrider and Suresh Srivastva (1986). It is a new paradigm that means to value and to recognize the positive nucleus of an organization or a system, seeking the strong points and talents of the individuals that belong to the organizations. From this point of view, the research group, which has the main objective to create, plan and design a library specialized in the natural stone (TinoStone Library), is working on knowing the users' information needs. To do that, the research group is applying a mix collecting data methods, such as questionnaires, interviews, etc. But the newness of applying $\mathrm{Al}$ is the way the interviews are done: potential users are invited to imagine, to design and to create in a collaborative way, "the ideal library" in order to share and implement knowledge intentional and actively. It is about a cooperative searching for the best of individuals and the best of organizations, avoiding negativeness and critics.
\end{abstract}

Keywords: Appreciative inquiry. User Studies. Enterprise Library.

- (a) PLAN-STONE: Planificación y análisis de usuarios de la biblioteca de la piedra natural

- (b) SIC-STONE: Diseño de un Sistema de Información sobre la Piedra; $y$,

- (c) CERI-STONE: Planificación de los espacios e instalaciones de la Piedra.

Centrándonos en el primer proyecto, es decir, el análisis y estudio de usuarios potenciales de la piedra natural, el Grupo de Investigación contempla tres tipos, agrupados en tres perfiles bien definidos: 
(a) Perfil de usuario empleado de Tino Stone, cuya composición y especialidad se conoce a través del organigrama funcional de la empresa y que incluye las áreas de compras, recursos humanos, ventas, tiendas, proyectos, postventa, $1+D$, marketing y nuevas instalaciones.

(b) Perfil de usuario cliente, que incluye a los prescriptores, es decir, arquitectos, aparejadores, diseñadores interioristas, promotores y constructores, cuyas recomendaciones como especialistas tienen influencia en la decisión de compra de otros usuarios.

(c) Perfil de usuario investigador de la piedra natural, que incluye científicos e investigadores especialistas en diferentes áreas de conocimiento, como química, geología, ciencia y tecnología de los materiales, ingeniería de la construcción, ingeniería mecánica, medioambiente, gestión de residuos, robótica y visión artificial, historia del arte, etc, cuyas líneas de investigación abordan el estudio de la piedra natural en alguna de sus vertientes.

\section{Objeto de la investigación}

Así, para conocer los hábitos y las necesidades de información de estos potenciales usuarios, con la finalidad de adecuar las colecciones, los espacios y los servicios, tanto físicos como virtuales, a las expectativas y necesidades informativas de los mismos, el Grupo de Investigación aplica una metodología mixta, que combina los métodos tradicionales de recogida de datos por medio de cuestionarios específicamente diseñados para ese fin, la cual hemos reflejado en el Anexo 1, complementada por la metodología que pretendemos dar a conocer aquí: la Investigación Apreciativa (IA), una metodología muy innovadora para promover el cambio en los sistemas sociales y organizacionales. De ahí, que la estén empleando organizaciones de diferentes niveles (multinacionales como Boeing, Nokia, Mac Donald; Organizaciones Gubernamentales: The U.S. Postal Service; Universidades: California-Berkeley, Case Western Reserve; ONGs: UNICEF, Cruz Roja; o en ciudades: Chicago, Nagaland -India-) y con objetivos muy diversos.

La biblioteca, considerada con un ente organizacional y, más concretamente, como una organización de aprendizaje, es ideal para su implementación, como reconoce Sullivan (2004). De hecho, ya han sido varias las bibliotecas de universidades norteamericanas las que están implementando esta metodología (lowa University, Brown University, George Washington University, University of Maryland, University of Cincinnati, etc.)

\section{Qué es la investigación apreciativa}

La Investigación Apreciativa (IA) es una metodología que se aplica en las organizaciones para promover el cambio, un cambio que se apoya en los puntos fuertes de los miembros de la organización, en lo que funciona, en lo positivo. Según Cooperrider (1986), promotor de esta teoría junto a sus colegas de la Case Western University:

Appreciative Inquiry is about the cooperative search for the best in people, their organizations, and the relevant world around them. In its broadest focus, it involves systematic discovery of what gives "life" to a living system when it is most alive, most effective, and most constructively capable in economic, ecological, and human terms.

Es una teoría del cambio con una visión sistémica del mismo. Para que el cambio sea efectivo tiene que incluir a toda la organización. Las decisiones verticales que se establecen en las organizaciones, donde se tienen en cuenta la individualidad y conocimiento de una jerarquía o grupo, es insuficiente para producir un cambio por todos y para todos.

De ahí que lo primero que nos sorprende de este enfoque metodológico reside en su principio colaborativo de que, entre todos, y de acuerdo con los sueños, aspiraciones y necesidades últimas de todos los involucrados en la organización (desde el director hasta el conserje) se construye la planificación y puesta en práctica de las estrategias acordadas para conseguir una organización mejor. Se trata de extraer lo mejor de cada uno, los talentos, las competencias, las habilidades..., que todos ellos lleven a obtener lo que se conoce como el núcleo positivo de la organización.

Un fenómeno similar en cuanto a los resultados es el que observamos en Internet con el movimiento actual de la Web 2.0, donde todos participan, colaboran, aportan y obtienen lo mejor del sistema, de la plataforma.

Es, por tanto, una búsqueda cooperativa de lo mejor que existe en la gente y en sus organizaciones. Cuando se trata de un cambio social, los participantes demandan su protagonismo, su reconocimiento y su aportación. Es un método que promueve el cambio mediante la creatividad, el conocimiento y, sobre todo, el espíritu colaborador.

¿Qué busca este enfoque? Precisamente, lo mejor de todos, que la positividad y el cambio lleven a algo mejor, una mejora infundida mediante el entusiasmo y optimismo. Esa es la clave. De ahí, que la metodología se realice mediante entrevistas abiertas y confeccionadas 
para estimular, para provocar. De la misma manera que suscita positividad, consecuentemente, las reacciones serán positivas 0 , al menos, no centradas en los problemas, el enfoque más implantado hasta ahora con las auditorías, control, sistemas de calidad, etc. (Whithey y Schau, 1998).

\section{Comparativa de la IA con el enfoque basado en la Resolución de problemas}

Para conocer mejor esta teoría del cambio, sería interesante presentar la comparación que hace Cooperrider y Srivastva (1987) del método tradicional o enfoque que resuelve a partir de la identificación de los problemas y el proceso que lleva la Investigación Apreciativa (Traducido por Varona, 2003, p.9) (Tabla I).

\begin{tabular}{ll}
\hline $\begin{array}{l}\text { Enfoque Resolución } \\
\text { Basado en Problemas }\end{array}$ & $\begin{array}{l}\text { Enfoque Investigación } \\
\text { Apreciativa }\end{array}$ \\
\hline $\begin{array}{l}\text { Identificación de } \\
\text { problemas }\end{array}$ & $\begin{array}{l}\text { Descubrir lo mejor que } \\
\text { existe }\end{array}$ \\
\hline Análisis de causas & $\begin{array}{l}\text { Imaginar lo que puede } \\
\text { llegar a ser }\end{array}$ \\
\hline $\begin{array}{l}\text { Plan de acción } \\
\text { (Tratamiento) }\end{array}$ & $\begin{array}{l}\text { Construir lo que debería } \\
\text { ser }\end{array}$ \\
\hline $\begin{array}{l}\text { Planteamiento básico: } \\
\text { Una organización es } \\
\text { un problema que hay } \\
\text { que solucionar }\end{array}$ & $\begin{array}{l}\text { Planteamiento básico: } \\
\text { un libro abierto que está } \\
\text { por escribirse }\end{array}$ \\
\hline
\end{tabular}

Tabla I. Comparativa enfoques Resolución de Problemas-IA.

Para que el cambio pueda producirse requiere, ante todo, un cambio de mentalidad particularmente de los directores o gestores de la organización, quienes van a hacer posible que su aplicación se lleve a cabo.

La IA no ignora los problemas, es más, los enfoca, pero desde una perspectiva diferente. Los resultados que se obtienen en la práctica de la IA algunas veces son exactamente iguales que los resultados obtenidos mediante el enfoque basado en problemas. Una gran diferencia entre ambas perspectivas es que en cualquier punto del proceso, la IA mantiene un alto grado de compromiso y energía entre los participantes, pues implica emocionalmente al personal en el proceso de cambio, algo que no sucede en los procesos basados en los problemas donde el propio personal se ve abocado a seguir unas meras instrucciones.

En cambio, otras veces, los resultados de la práctica de la IA no tienen nada que ver con los resultados del enfoque basado en problemas. Las ideas brillantes que a menudo se generan con la IA son de tal alcance que hubieran sido impensables de lograr mediante el otro enfoque.

\section{Ideas que destacan en la práctica de la IA como enfoque metodológico de cambio}

A continuación, resaltamos algunos conceptos interesantes que vienen a definir esta metodología de cambio: (a) Cambiar la manera de ser: es aprender a ver el "vaso medio lleno" en lugar del "vaso medio vacío". (b) Cambiar la manera de ver: sabiendo reconocer lo bueno en lugar de lo malo en todo lo que nos rodea. (c) Cambiar la manera de juzgar: apoyarse en lo positivo que existe a nuestro alrededor en lugar de en lo negativo. (d) Cambiar la manera de conversar dialogar-: usando un lenguaje positivo en lugar de negativo. (e) Cambiar la manera de aprender: del éxito en lugar del fracaso. (f) Creer que el cambio positivo es posible. (g) Creer en el poder transformador de la conversación como recurso para cambiar y crear nuestra visión de la organización y de la comunicación (Varona, 2003).

\section{Cómo se aplica la metodología de la Investigación Apreciativa}

El proceso de la Investigación Apreciativa es muy flexible en relación a los participantes y al tiempo invertido. Es conveniente que cuantas más personas de la organización estén implicadas, mejor, pues promueve un alto nivel de compromiso y energía de los participantes. En cuanto al tiempo que se invierte en llevar a cabo un proceso de IA, dependerá del grupo y de los objetivos del mismo: puede durar desde un día hasta meses.

No obstante, las dos modalidades que con más frecuencia se emplean son: (a) La modalidad cumbre que consiste en que la organización entera dedica un tiempo de cuatro días para completar todo el proceso; y (b) La modalidad movimiento social que inicia el método en un grupo determinado de la organización dirigido por un facilitador de IA quien define y explica cómo se va a desarrollar el proceso, cuánto tiempo se va a emplear y quiénes van a participar (Varona, 2003).

Las fases de aplicación que nos ofrece Cooperrider (1986) son cuatro: las conocidas cuatro D (Discovery, Dream, Design y Destiny), que más tarde se convertirán en cinco D (Define) (Cooperrider, 2000). 
En este sentido, vamos a describir las fases $y$, paralelamente, detallar la aplicación de las mismas en nuestra investigación para el análisis de las necesidades de información de los potenciales usuarios de la biblioteca TINO Stone.

\subsection{Fase Definir (Define)}

En esta fase se define cómo se va a hacer el proceso de IA. Es un proceso, como venimos indicando, flexible, pues puede emplearse con grupos de todos los tamaños y con cronogramas muy variados, según convenga a todos los miembros de la organización.

\section{Definir Biblioteca Tino Stone:}

(a) Se parte de una comisión compuesta por dos investigadores que identifican el grupo de usuarios (empleados, clientes e investigadores), y que actúan como facilitadores de la IA; seleccionan una muestra representativa de cada grupo y realizan unas entrevistas previamente diseñadas empleando un lenguaje positivo y "provocativo". Lo que se conoce como "Entrevistas provocativas". (b) El tiempo estimado es de dos meses (mayo-junio, 2007). (c) Misión de la Biblioteca Tino: Organizar y proporcionar servicios y recursos de información especializados en piedra natural que contribuyan a convertir a Tino Stone en líder y referente mundial en lo que se refiere el proceso de negocio, la investigación y la cultura de la piedra natural. (d) Visión de la Biblioteca Tino: Ser la biblioteca referente mundial de la Piedra Natural. (e) Valores: servicio, respecto, comunicación, colaboración, diversidad, responsabilidad y ética (University Libraries: University of Cincinnati, 2003).

\subsection{Fase Descubrir (Discovery)}

En esta fase se trata de movilizar a la organización persiguiendo descubrir lo que la gente valora, espera y desea de ese sistema u organización. Se trata de investigar qué es lo que funciona bien y por qué. A través de la Entrevista Apreciativa, se conocen las mejores experiencias, las ideas más valiosas y el futuro deseado de los participantes. Las preguntas suelen responder al mismo esquema "provocativo". Para conocer más sobre cómo formular estas preguntas, los autores de la IA han editado Encyclopedia of Positive Questions, Vol. I: Using Al to Bring Out the Best in Your Organization (2001) donde se recoge un elenco de preguntas para aplicar en esta fase de descubrimiento.

\section{Descubrir en la Biblioteca Tino Stone}

(a) La comisión IA entrevista a grupos representativos de los futuros potenciales usuarios de la Biblioteca Tino Stone. (b) Se diseña la entrevista con las siguientes preguntas abiertas de carácter provocativo, tales como (Anexo II):

(b.1) Describe una experiencia positiva como usuario de biblioteca

(b.2) ¿Qué factores crees que contribuyeron al éxito de esa experiencia?

(b.3) ¿Qué es lo que más valoras de tu experiencia en una biblioteca? ¿Por qué?

(b.4) Si tuvieras una varita mágica, ¿Cuáles son los tres deseos que mejorarías en una biblioteca para el futuro?

(c) La comisión IA, una vez que ha recopilado la información de las muestras representativas, analiza los contenidos para determinar los temas comunes que giran en torno a las preguntas provocativas formuladas.

\subsection{Fase Soñar (Dream)}

Los participantes imaginan su deseo de futuro y le dan forma. Un sueño que puede estar expresado de formas diferentes: por escrito, o expresado en un sketch, una poesía, un baile, etc. Se le ofrece la posibilidad al participante de que exprese su sueño del objetivo como desee y que ponga en práctica su creatividad. Las formas más comunes para expresar este deseo son mediante un texto escrito o una expresión artística.

En esta fase se emplean dos escalas para valorar la importancia de las proposiciones:

(a) Una escala que vaya de Muy importante a Poco importante para valorar las proposiciones: así por ejemplo, muy importante se considera el valor que el participante otorga al espacio de la biblioteca; poco importante se considera la existencia de un diccionario en una lengua muy minoritaria, como el Alsaciano y poco relacionado con los países donde se trabaja con la Piedra Natural (ya sea por explotación, tratamiento y/o comercialización).

(b) Otra escala que identifique la existencia o no de una proposición provocativa: Sí, No, De alguna Manera.

\section{Soñar en la Biblioteca Tino Stone}

(a) La comisión IA elige la expresión escrita para que el participante exprese su sueño de Biblioteca de la Piedra Natural. Para ello, aprovecha el mismo intervalo de tiempo de la entrevista de la fase Descubrir.

(b) Las proposiciones provocativas de esta fase giran en torno a los servicios, los espacios, los fondos y al personal (Anexo II). 
(b.1) ¿Cómo te gustaría trabajar en una biblioteca? Nos referimos al espacio, a la sala de lectura, de consulta (condiciones medio-ambientales)

(b.2) ¿Cómo te imaginas el edificio físico de una biblioteca ideal? Exprésalo mediante frases, calificativos, dibujos, esquemas...

\subsection{Fase Diseñar (Design)}

Para que ese sueño expresado por los participantes se cumpla, el grupo -en nuestro caso la comisión IA - crea las posibilidades viables para llevar a cabo los objetivos propuestos; es decir, cómo se va a ejecutar para lograr la realización de los sueños.

En esta fase es imprescindible realizar un análisis de recursos para identificar aquellos que existen y los que se van a necesitar para ejecutar el programa de acción.

\section{Fase Diseñar en la Biblioteca Tino Stone}

El análisis de los resultados obtenidos en las dos fases anteriores -Discovery y Dreampermitirá a la comisión IA proponer una preplanificación de servicios, espacios, colecciones y personal de la Biblioteca de la Piedra Natural Tino Stone, sumando las expectativas y deseos de los diferentes grupos de usuarios.

A modo de ejemplo en torno al personal bibliotecario, Sullivan (2004) nos indica cómo trabajar juntos en la construcción de la organización de esa biblioteca imaginada en la fase Soñar. El personal realizará una descripción detallada del propio trabajo de diseño, estructura, sistemas, cultura y entorno de trabajo que han deseado.

\subsection{Fase Ejecutar (Destiny)}

El grupo, o en los que se haya delegado, diseña la planificación real y final para implementar los primeros pasos que se hayan decidido. Opcionalmente, en cualquier etapa del proceso, el grupo puede volver a la fase de "Discovery" descubrir-y hacer una mini-IA para impulsar el proyecto general.

En esta etapa de ejecución, la implicación personal y organizacional ante el cambio es total.

\section{Fase Ejecutar en la Biblioteca Tino Stone}

En esta última etapa la Comisión IA de la Biblioteca Tino Stone diseñará la planificación final de este proceso IA cuyo objetivo ha sido conocer las necesidades y expectativas de información de los potenciales usuarios de esta biblioteca especializada.

\section{Principios de aplicación de la Investigación Apreciativa}

La consultora M. Sullivan (2004) ha recopilado un conjunto de principios procedentes de su práctica en Investigación Apreciativa en Organizaciones, particularmente, en organizaciones de aprendizaje. Al considerar a la biblioteca en este marco, nos ofrece una serie de principios aplicables a la misma para llevar a cabo una IA: (a) Reconoce a las bibliotecas como organismos vivos, sistemas orgánicos. El personal de cualquier nivel, da vida a la organización bibliotecaria. (b) El comportamiento del presente tiene una gran influencia en el futuro que se anticipa. Los directivos tienen la oportunidad de producir el cambio comprometiendo al personal bibliotecario en el ciclo de las $4 \mathrm{D}$ y centrarse en las posibilidades positivas del futuro. (c) Ambos enfoques son procesos generativos. "Avanzar aprendiendo es algo emocionante", libera la capacidad del personal, y capacita a la organización para ser mejor. (d) Es muy importante hacer preguntas eficaces y relevantes. (e) Los directivos juegan un papel muy importante al dirigir positivamente el proceso de IA y el proceso de aprendizaje. (f) El éxito continuado proviene del compromiso del personal y del aprecio de su esfuerzo e implicación. (g) La gente tiene, tanto de forma individual como colectiva, habilidades, talentos y contribuciones que aportar a la vida de la biblioteca. (h) Cualquier esfuerzo de cambio a largo plazo requiere un compromiso para el continuo aprendizaje, crecimiento y desarrollo de la organización de los grupos y equipos y de todos los individuos.

\section{Beneficios de la IA}

La aplicación de esta innovadora metodología en las organizaciones, aporta una serie de beneficios que hasta ahora no se ha conseguido con otras metodologías de gestión de cambio en las organizaciones. Esto es así porque: (a) Los resultados de la IA crecen de la experiencia colectiva, de la sabiduría y de los recursos de los participantes. Es una obra colectiva trabajada conjunta y activamente. (b) La IA es democrática y valora la diversidad en una organización. Este respeto a la diversidad hace a la organización más rica, con miembros más informados y más motivados para trabajar colectivamente y obtener beneficios para todos. (c) La IA desde el mismo momento en que se inicia genera cambio, pues se caracteriza por ser provocativa, alternativa, y motivadora de energía productiva, contrariamente a lo que sucede con el método enfocado a la resolución del problema, que produce un efecto de bloqueo en las mentes de los participantes. (d) La IA reutili- 
za experiencias propias positivas del grupo participante para ser empleadas en el futuro. (e) Es sostenible y realista puesto que las soluciones diseñadas por la IA crecen desde una experiencia colectiva y no basadas en principios abstractos o procedentes de expertos externos.

\section{Conclusiones}

Toda IA es única. La nuestra, aplicada en el proceso de planificación de una biblioteca especializada y, más concretamente, en la fase de análisis y estudio de usuarios, nos ha permitido obtener datos sorprendentes. El importante papel que ejerce el lenguaje -lenguaje provocativo- utilizado en las fases de Discovery y Dream ha motivado que los participantes liberen su creatividad y se obtengan productos imaginativos realizables y reales, quizás impensables mediante una metodología tradicional.

Vayan aquí, a modo de ejemplo, algunas de las ideas proporcionadas por los usuarios y con potencialidad para hacerse factibles: (a) talleres experimentales para conocer las diferentes utilizaciones de la piedra; (b) personal con una estética y actitud agradable, etc.; (c) servicio de digitalización de documentos "para llevar" (take away documentary service); (d) que la biblioteca ofrezca un espacio físico y virtual de encuentro entre profesionales de la piedra; (e) grandes y confortables sofás de cuero, a modo de salón de té, con amplios espacios luminosos y libres de ruidos; (f) que el bibliotecario prácticamente no exista.

Utilizar la IA permite tener una perspectiva diferente a la metodología tradicional, al tener como punto de partida los valores positivos y fuertes de la organización. La IA no oculta los problemas sino que los aborda desde otra perspectiva. Con la IA se motiva e implica a todos los participantes en el diseño y planificación del objeto, en este caso, la biblioteca de la Piedra Natural.

\section{Referencias}

ANECA. 2005. Guía para la Evaluación de bibliotecas Universitarias. Madrid, Aneca, 2005.

The Appreciative Inquiry Commons [en línea]. Cleveland $(\mathrm{OH})$ : Weatherhead School of Management- Case Western Reserve University. Disponible en http://appreciative inquiry.case.edu/default.cfm (consulta junio 2007)

Cooperrider, D.L. (1986) Appreciative Inquiry: Toward a Methodology for Understanding and Enhancing Organizational Innovation (Theory, Social Participation). Case Western Reserve, 1986.

Cooperrider, D.L. et al. (2000). Appreciative Inquiry: Rethinking Human Organization Toward a Positive Theory of Change. Champaign, IL: Stipes, 2000.
Whitney, D.L., Cooperrider, D.L., Kaplin, B.S. TrostenBloom, A. (2001). Encyclopedia of Positive Questions, Vol. I: Using Al to Bring Out the Best in Your Organization. Cleveland, OH: Crown Custom Publishing, 2001.

Cornella, A. 1994. The Role of Information Service in an Information-Intensive Environment. Barcelona: Esade, 1994 (Papers Esade, $n^{\circ}$ 132).

Ferrer Torrens, Adelaida y Rey Martín, Carina. Aplicación del Libqual en el CRAI de la Universidad de Barcelona [documento en pdf]. Infogestión : 9as Jornadas Españolas de Documentación: Madrid, 14 y 15 de abril de 2005, Madrid: FESABID, 2005. Disponible en http://www. recercat.net/bitstream/2072/1497/1/libqual.pdf (consulta junio 2007)

Fundación del Centro Tecnológico Andaluz sobre la Piedra (CTAP) [en línea]. Disponible en http://www.ctap.es/ (consulta junio 2007).

Nicholas, D. 2000. Assessing Information Needs: Tools and Techniques. $2^{\mathrm{a}}$ ed. Londres: Aslib, 2000.

Observatorio del Mercado de la Piedra Natural [documento en pdf]. Aidico-Instituto Tecnológico de la Construcción, 2003. Disponible en http://om pn.aidco.es/php_lib/fichero _descarga. php?oid $=1171611 \&$ nombre $=$ Memoria\& $\&$ tipo $=p$ $\overline{\mathrm{df}}$ (consulta mayo 2007).

Parasuraman, A., Zeithaml, V.A. y Berry, L.L. 1988. SERVQUAL: A multiple item scale for measuring consumer perceptions of quality service. // Journal of Retailing. 64:1 (1988) 12-40.

Pinto, M. et al. 2006. Diseño de la herramienta electrónica BiQual para la evaluación y mejora continúa de sistemas de información. Aplicación a ciencia y tecnología, SEC2002-03092, Ministerio de Ciencia y Tecnología, 2003-2006.

REBIUN. Plan Estratégico 2003-2006. Disponible en http://www.crue.org/rebiun/PlanEstrategico-Definicionbi blioteca.pdf (consulta mayo 2007),

Stonereport: Actualidades para su empresa. // NürnbergMesse- Stein Journal. http://www.stonereport. com/ihtm/start-s.htm (consulta junio 2007).

Strategic Plan: 2003-2005. [documento pdf]. Cincinnati: University Libraries- University of Cincinnati, February 2003. http://www.libraries.uc.edu/information/planning/ strategicplan.pdf (Consulta junio 2007).

Sullivan, M. (2004) The promise of Appreciative Inquiry in Library Organizations. Library Trends, vol. 53, $n^{\circ} 1$, pp. 218-229.

TINO. Dossier de prensa. Diciembre 2006 [documento en pdf]. // Goodwill Comunicación, 2006. http://www.good will.es/cliente. php?cID=52 (consulta junio 2007).

TINO Stone Group invertirá 30 millones de euros en la creación de un gran parque industrial de la piedra en Loja (Granada). 14/05/2007. http://www.goodwill.es/noti cia.php?nID=234 (Consulta 11/05/2007)

Whitney, D. And Schau, C (1998). Appreciative inquiry. An innovative process for organization change. // Employment relations today. (Spring 1998) 11-20. 


\section{Apéndice}

\section{Anexo 1: Metodología de los cuestionarios}

Los tres modelos de cuestionarios diseñados, uno por cada perfil de usuarios, para ser distribuidos de forma electrónica, están estructurados en varios apartados: datos institucionales, líneas de investigación y áreas de trabajo específicas, comportamientos y usos de información, expectativas y necesidades en cuanto a colecciones, espacios y servicios, y valor de la biblioteca de la piedra.

Para diseñar los apartados de los cuestionarios referentes al ámbito específico de la piedra natural, como por ejemplo las líneas de investigación y las áreas de trabajo, la procedencia y tipo de las fuentes de información externa o las necesidades de información específicas para este ámbito, nos hemos valido de informes especializados procedentes de fuentes de información diversas, como el Observatorio del Mercado de la Piedra Natural, el Dossier de prensa de la empresa Tino (Tino, 2006), la revista Stonereport, y la Fundación del Centro Tecnológico Andaluz sobre la Piedra (CTAP).

Respecto a los apartados relativos a comportamientos, expectativas y necesidades en cuanto a las colecciones, los servicios y los espacios de la biblioteca de la piedra, contamos en este punto con un nutrido elenco de experiencias llevadas a cabo recientemente en bibliotecas universitarias españolas, como los Planes de calidad en Biblioteca de la ANECA (2005) o el Plan Estratégico de REBIUN, que nos han proporcionado un abundante corpus teórico y práctico, procedentes del ámbito de la gestión de calidad de las bibliotecas, y basadas en la percepción de los usuarios sobre la calidad del servicio, que expresan sus opiniones acerca de diferentes dimensiones: prestación de servicios, accesibilidad, funcionalidad, espacios, etc., para finalmente obtener datos y conseguir mejoras. En este sentido, existen una serie de herramientas y metodologías de diagnóstico específicamente diseñadas para la evaluación integral de los diferentes aspectos del servicio y la gestión de estas bibliotecas. Entre ellas, Libqual ${ }^{+T M}$, un método que "permite observar la calidad de los servicios a partir de los datos proporcionados por los usuarios sobre sus percepciones respecto a los servicios proporcionados en las unidades de información" (Ferrer y Rey, 2005), basado en el cuestionario Servqual (Parasuraman, Zeithaml y Berry, 1988). Así como una metodología más reciente, también desarrollada bajo el enfoque de la voz de los usuarios y centrada en las bibliotecas universitarias de ciencia y tecno- logía como BIQUAL, considerada tanto una herramienta de diagnóstico de calidad del servicio como un instrumento idóneo para conocer nuevas tendencias en el uso de la información.

Así pues, decidimos utilizar el modelo BIQUAL (Pinto, 2006), que mejora y complementa los modelos Libqual y Servqual en los que se basa.

La decisión de utilizar esta herramienta se justifica por las siguientes razones:

1. Es una herramienta centrada en conocer la opinión de los usuarios respecto a diferentes dimensiones sobre calidad de servicios de bibliotecas: accesibilidad de los servicios, funcionalidad de las prestaciones, comunicación de la biblioteca con los usuarios, usabilidad de los servicios y servicios de información innovadores y de última tendencia.

2. Los resultados obtenidos al aplicar esta herramienta identifican comportamientos de los usuarios

3. Se trata de una herramienta diseñada específicamente para bibliotecas especializadas en ciencia y tecnología, una de las principales líneas de investigación y actuación en el ámbito de la piedra natural.

4. Se trata de una herramienta diseñada para establecer diagnósticos sobre calidad de servicios para bibliotecas, pero también para obtener información decisiva para el diseño de servicios y productos en otro tipo de bibliotecas especializadas.

\section{Anexo II: Entrevistas de la fase Discovery de} Bibliotecas

- Describe una experiencia positiva con una biblioteca (desde siempre, desde que empezaste tus estudios, hoy por hoy con las nuevas tecnologías)

- ¿Qúe factores crees que contribuyeron al éxito de esa experiencia?

- ¿Qué es lo que más valoras de tu experiencia en una biblioteca? ¿Por qué?

- Imagínate que tienes una varita mágica y te permite que se cumplan tres deseos en torno a la biblioteca. ¿Cuáles son los tres deseos para mejorar una biblioteca para el futuro e ir en la buena dirección?

- ¿Cómo te imaginas el edificio físico de una biblioteca ideal? Exprésalo mediante frases, calificativos, dibujos, esquemas... 
- ¿Qué fondos te gustaría encontrar en una biblioteca? ¿Todos los libros? ¿Enciclopedias? ¿Bibliografías? ¿Revistas impresas? ¿Revistas electrónicas? ¿Material audiovisual? ¿Cartografía? ¿Bases de datos especializadas?...

- ¿Y si pudieras acceder a los fondos de la biblioteca por Internet? ¿Qué te gustaría encontrar?

- ¿Cómo te gustaría trabajar en la biblioteca? Nos referimos al espacio, a la sala de lectura, de consulta en la biblioteca (condiciones medioambientales)

- ¿Qué servicios te gustaría que te proporcionase la biblioteca ideal y que te faciliten tu trabajo? Por ejemplo, proporcionarte por email información especializada y personalizada, o seleccionarte información sobre revistas especializadas...

- ¿Cómo piensas que puedes acudir a una biblioteca y saber manejar con soltura el catálogo automatizado y poder encontrar lo que buscas rápida y fácilmente? 